\title{
A physically-motivated model describing the dynamic interactions between residual limb and socket in lower limb prostheses
}

DOI 10.1515/cdbme-2017-0004

\begin{abstract}
The amputee's well-being and mobility are distinclty related to socket fit and resulting biomechanical interaction between residual limb and prosthetic socket. Understanding the dynamic interactions at the interface may lead to new socket standards. This paper introduces a physically-motivated reduced model of the interface, describing the dynamic interactions between residual limb and prosthetic socket. The model allows to investigate the sensitivity to changes of specific parameters in an isolated matter. A simulation study shows how stress distribution changes if friction coefficients are varied which might advance liner design.
\end{abstract}

Keywords: lower limb prostheses; modelling; residual limbsocket interaction; socket fit.

\section{Introduction}

Socket fit is crucial for the well-being and mobility of amputees [1]. Being the interface between human and prosthesis, the socket provides stability and control of the entire device. Quantitative knowledge about biomechanical interactions at the interface, particularly during gait, is rare [2]. A possibility for gaining a better understanding of the interaction between prosthesis and residual limb is computational modeling and simulation. In the literature, mainly FE-models can be found [2]. Yet, these models are

\footnotetext{
*Corresponding author: Veronika Noll, Institute for Mechatronic Systems in Mechanical Engineering, TU Darmstadt, Darmstadt, Germany, E-mail: noll@ims.tu-darmstadt.de

Niclas Eschner: wbk Institut für Produktionstechnik, Karlsruher Institut für Technologie, Karlsruhe, Germany

Christian Schumacher: Lauflabor Locomotion Laboratory, Institute of Sport Science, TU Darmstadt, Darmstadt, Germany

Philipp Beckerle and Stephan Rinderknecht: Institute for

Mechatronic Systems in Mechanical Engineering, TU Darmstadt, Darmstadt, Germany
}

currently not able to describe the dynamic interactions due to nonlinear boundary conditions.

The development of a physically-motivated reduced model of the interface describing the dynamic interactions between residual limb and prosthetic socket is needed. With this model, experimental studies concerning comfort, stability, and control can be assisted by an integrated approach of model and experiment. E.g., simulative parameter studies can lead to new experimental trial designs.

This paper presents model design and implementation, including parameter configuration, optimization criteria and evaluation of model performance. Furthermore, an exemplary biomechanical analysis is discussed. Subsequently, concluding remarks and an outlook on future work are given.

\section{Model design}

The synthesized model is visualized in Figure 1. It consists of the socket system (PTB socket with shuttle lock), and the residual limb (soft tissue and bone element) with liner. To account for pliant soft-tissue dynamics, the model integrates eight contact points distributed equally around the liner surface. Figure 1 shows the rotationally symmetric model as cross-section with indicated positions of the other contact points (cf. Liner). Bone and socket elements are modeled as rigid bodies. Liner deformation is neglected, while soft tissue mechanics are implemented as KELVIN models with progressive spring characteristics. The shuttle-lock system is implemented as tractionforce-limited KeLVIN model. The occurrence of slippage between liner and socket is described by the integration of a STRIBECK friction model.

\subsection{Parameter configuration}

The static friction coefficient between liner and socket is set in accordance with [3, 4]. As suggested in [5], the dynamic 


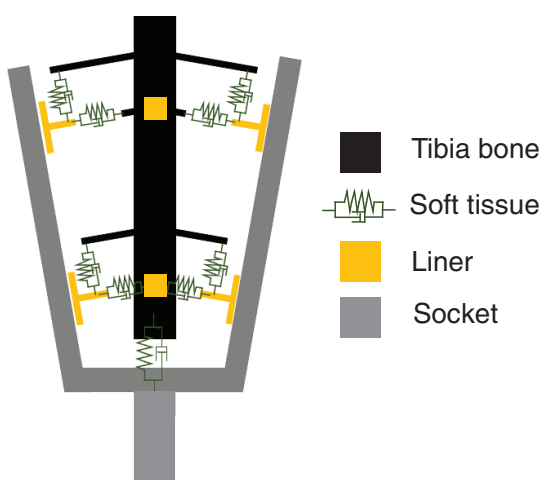

Figure 1: Abstraction of residual limb and prosthetic socket in model.

friction coefficient is set to $70 \%$ of the static one. Initial values for the spring characteristics on the normal plane $\left(c_{i}\right)$ are formulated by the progressive Young's modulus described in [6], using Hooke's law:

$$
c_{i}=\frac{E_{i} A}{l} .
$$

$(A)$ denotes the area and $(l)$ the width of the considered residual limb region. Analogously, the spring characteristics for the shear plane $c_{\mathrm{q} i}$ are calculated via

$$
c_{\mathrm{q} i}=\frac{E_{i} A}{2(1+v) l}
$$

with Poisson's ratio $(v)$ set to 0.5 to meet the incompressibility of soft tissue mechanics (cf. [7, 8]). Due to a lack of information in the literature, damping coefficients are determined iteratively.

Model parameters concerning the geometry of the socket and the residual limb are taken from an existing data set [9] and simplified to a cone shape. Due to missing information about residual limb composition, a CT scan from the literature [10] is used to approximate relevant parameters, e.g., width of residual limb region $(l)$. Wobbling mass is estimated based on cone volume and the assumption that $50 \%$ of the identified volume (density $\rho=1000 \mathrm{~kg} / \mathrm{m}^{3}$ ) is accountable to soft tissue wobbling mass. In this first study, the mass and lower limb surface are distributed equally between the eight contact points. Values of the discussed parameters are summarized in Table 1.

\subsection{Implementation and optimization}

The model is implemented in SimMEchanics of MATLAB SimULINK. For simulation, experimentally measured loads (special force and torque measurement adapter, integrated into prosthetic alignment [9]) are applied to the shank tube
Table 1: Summary of values of discussed model parameters.

\begin{tabular}{lll}
\hline Parameter and explanation & Value \\
\hline$\mu_{0}$ & Static friction coefficient & 0.5 \\
$\mu_{d}$ & Dynamic friction coefficient & 0.35 \\
$E_{10}$ & Young's modulus for deformations $<10 \%$ & $11.2 \mathrm{kPa}$ \\
$E_{20}$ & Young's modulus for deformations $<20 \%$ & $25.6 \mathrm{kPa}$ \\
$E_{30}$ & Young's modulus for deformations $<30 \%$ & $40 \mathrm{kPa}$ \\
$E_{31}$ & Young's modulus for deformations $\geq 30 \%$ & $54.4 \mathrm{kPa}$ \\
$l$ & Width of residual limb region & $0.03 \mathrm{~m}$ \\
$v$ & Poisson's ratio & 0.5 \\
$A$ & Area assigned to each contact point & $0.0004 \mathrm{~m}^{2}$ \\
$m$ & Mass assigned to each contact point & $0.03 \mathrm{~kg}$ \\
\hline
\end{tabular}

at the distal end of the socket. As a model simplification, only loads in the sagittal plane, i.e., forces in anteroposterior and proximodistal direction as well as torque around mediolateral axis, are considered. The tibia bone is affixed.

\subsubsection{Optimization criteria}

The developed model predicts pressure and shear stress at the contact points. Further, it anticipates relative movement between residual limb and prosthesis for the contact points as well as the bone element. The proposed model is optimized considering experimental values taken from the literature. To the best of the authors' knowledge, shear stress at the interface is not discussed experimentally up to now.

Considering pressure distributions at the interface, a variety of experimental values can be found in the literature (e.g., [11-14]). However, experimental data collection differs regarding the number of subjects and used sensor type. Particularly the work of [15] shows, the dependency of the values to these variations in the experimental protocol. A general statement considering pressure distributions as formulated by [16] is disproved and cannot be renewed, due to contradicting values in the literature. Nevertheless, a range of pressure values ( $<70$ to $120 \mathrm{kPa}$ ) as well as gaitcycle dependent appearance (similar to vertical ground reaction forces) can be defined as optimization criteria.

The relative movement at the interface can be categorized into three distinguishable relative movements. Depending on residual limb (RL) area and element moving relative to the prosthetic socket: distal end of RL, RL (or liner) surface, and tibia bone. Table 2 summarizes values from the literature, which serve as points of reference considering optimization criteria. All listed values are for PTB sockets with shuttle-lock system. Although experimental values differ depending on analyzed gait and subject 
Table 2: Summary of experimental data from the literature considering different types of relative movements.

\begin{tabular}{|c|c|c|}
\hline \multicolumn{2}{|c|}{ Reference and value } & Experimental constraints \\
\hline \multicolumn{3}{|c|}{ Distal end of RL to socket } \\
\hline [17] & $<4 \mathrm{~cm}$ & 1 Subj., Photoelectric and dynamic \\
\hline [18] & $<3.1 \mathrm{~cm}$ & 20 Subj., Potentiometer and dynamic \\
\hline \multicolumn{3}{|c|}{ RL or liner surface to socket } \\
\hline [19] & $<1.6 \mathrm{~cm}$ & 20 Subj., Not mentioned and dynamic \\
\hline [9] & $<0.5 \mathrm{~cm}$ & 1 Subj., Motion capture and dynamic \\
\hline \multicolumn{3}{|c|}{ Tibia bone to socket } \\
\hline [20] & $<3.5 \mathrm{~cm}$ & 7 Subj., X-ray and four static conditions \\
\hline [21] & $<0.5 \mathrm{~cm}$ & 1 Subj., X-ray and three static conditions \\
\hline [22] & $<1 \mathrm{~cm}$ & 3 Subj., Cineradiography and dynamic \\
\hline
\end{tabular}

condition, a value range for the distinguished forms of relative movement can be determined as given in Table 2 .

\subsubsection{Parameter changes due to optimization}

The optimization process reveals that pressure and relative movement values are higher than the identified ranges allow. In comparison to FE-models in the literature, the Young's modulus in the synthesized model are low. The values for $E_{i}$ described in Table 1 are increased by $50 \%$. An adaptation of residual limb geometry parameters (lowering width values) would lead to similar results.

\subsection{Evaluation of model performance}

The discussed optimization criteria can be satisfied with changes to the previously defined soft tissue characteristics. A full validation is aspect of future work. Nevertheless, due to the physical motivation of the model design and its parameters, it can be used to analyze biomechanical changes due to parameter variations at the interface. Due to the reduced complexity of the model, it has a simulation duration of five seconds for two gait cycles. Thus, permitting to assess the effects of dynamical interactions at the interface.

\section{Biomechanical analysis}

As simulation input data, the loads measured in the prosthetic structure during gait $(0.85 \mathrm{~m} / \mathrm{s})$ on a treadmill of one trans tibial amputee [9] is used. The mean of approximately 60 gait cycles is applied to generate a load input vector consisting of 100 equivalent gait cycles.

To qualitatively assess interface behavior due to parameter variations, their influence on overall (mean) pressure

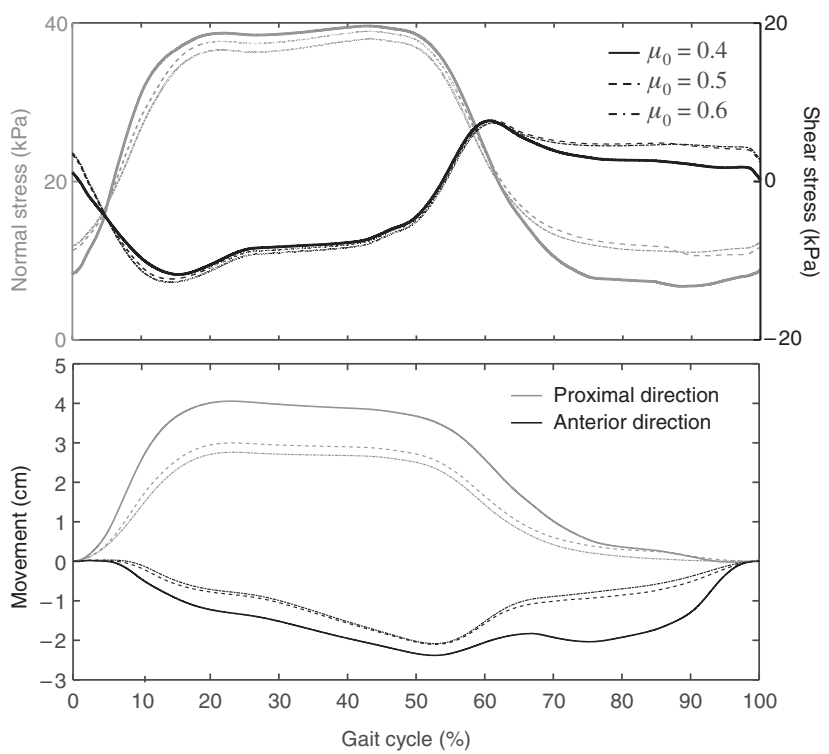

Figure 2: The effect of friction coefficient variation on stress distribution (top) and movement (bottom).

and shear stresses as well as movement of distal end of tibia relative to the socket, is presented.

\subsection{Results}

Exemplary, the effect of a change in the friction coefficient $\mu$ is shown in Figure 2. With lower $\mu$ the normal stresses at the interface increase while shear stresses decrease in stance phase. This is accompanied by larger relative movements of the tibia bone within the socket.

\subsection{Discussion}

Simulation results suggest that the force transmission during stance phase shifts from shear to normal forces for lower friction coefficients $\mu$. Considering liner materials with different friction coefficients might be an option for manipulating stress condition at the interface. In addition, the use of elastic strain gauges to measure interface stresses has to be reconsidered, when accompanied by changes to the ordinary friction coefficient.

\section{Conclusion and outlook}

The proposed model predicts pressure and shear stress as well as relative movement between residual limb and prosthesis. Compared to measurement values from the literature, simulation results are in a plausible range. Due to a lack of measurement data, a full validation has not taken 
place yet. Nevertheless, the proposed simulation model yields information that can contribute to the understanding of dynamic interaction between prosthesis and residual limb during gait with respect to individual parameters, e.g. frictions coefficients. These model indications may be used for experimental trial design.

After a validation of the model for different experimental data sets, future work may include refined local parameter adaptations and the analysis of their effect on the dynamic interaction at the residual limb-socket interface.

\section{Author's Statement}

Research funding: The IGF project 18873 N/2 of the Forschungsvereinigung für Messtechnik, Sensorik und Medizintechnik e.V. Dresden was funded by the AiF within the framework of the program for the promotion of industrial joint research (IGF) through the Federal Ministry of Economics and Energy due to a decision of the German Bundestag. Conflict of interest: Authors state no conflict of interest. Informed consent: Informed consent has been obtained from all individuals included in this study. Ethical approval: The research related to human use complies with all the relevant national regulations, institutional policies and was performed in accordance with the tenets of the Helsinki Declaration, and has been approved by the authors' institutional review board or equivalent committee.

\section{References}

[1] Christ O, Jokisch M, Preller J, Beckerle P, Wojtusch J, Rinderknecht S, et al. User-centered prosthetic development: comprehension of Amputees' needs. In: Biomedical Engineering 57.S1 2012. p. 1098-101.

[2] Mak AF, Zhang M, Boone DA. State-of-the-art research in lower-limb prosthetic biomechanics-socket interface: a review. J Rehabil Res Dev. 2001;38:161-74.

[3] Sanders JE, Greve JM, Mitchell SB, Zachariah SG. Material properties of commonlyused interface materials and their static coefficients of friction with skin and socks. J Rehabil Res Dev. 1998;35:161-76.

[4] Zhang M, Mak AF. In vivo friction properties of human skin. Prosthet Orthot Int. 1999;23:135-41.

[5] Müller R. Klassische Mechanik: Vom Weitsprung zum Marsflug. [Elektronische Ressource]. Berlin: de Gruyter; 2009. - URL http://dx.doi.org/10.1515/9783110213164.

[6] Zheng Y, Mak AF, Lue B. Objective assessment of limb tissue elasticity: development of a manual indentation procedure. J Rehabil Res Dev. 1999;36:71-85.

[7] Zhang M, Mak AF. A finite element analysis of the load transfer between an above-knee residual limb and its prosthetic socket-roles of interface friction and distal-end boundary conditions. IEEE Trans Rehabil Eng. 1996;4:337-46.

[8] Zhang M, Turner-Smith AR, Roberts VC, Tanner A. Frictional action at lower limb/prosthetic socket interface. Med Eng Phys. 1996;18:207-14.

[9] Noll V, Wojtusch J, Schuy J, Grimmer M, Beckerle P, Rinderknecht $S$. Measurement of biomechanical interactions at the stump-socket interface in lower limb prostheses. Conf Proc IEEE Eng Med Biol Soc. 2015;2015:5517-20.

[10] Portnoy S, Yizhar Z, Shabshin N, Itzchak Y, Kristal A, DotanMarom Y, et al. Internal mechanical conditions in the soft tissues of a residual limb of a trans-tibial amputee. J Biomech. 2008;41:1897-909.

[11] Ali S, Osman NA, Mortaza N, Eshraghi A, Gholizadeh H, Wan Abas WA. Clinical investigation of the interface pressure in the trans-tibial socket with Dermo and Seal-In X5 liner during walking and their effect on patient satisfaction. Clin Biomech (Bristol, Avon). 2012;27:943-8.

[12] Convery P, Buis AW. Socket/stump interface dynamic pressure distributions recorded during the prosthetic stance phase of gait of a trans-tibial amputee wearing a hydrocast socket. Prosthet Orthot Int. 1999;23:107-12.

[13] Dumbleton T, Buis AW, McFadyen A, McHugh BF, McKay G, Murray KD, et al. Dynamic interface pressure distributions of two transtibial prosthetic socket concepts. J Rehabil Res Dev. 2009;46:405-15.

[14] Eshraghi A, Osman NAA, Gholizadeh H, Ali S, Sævarsson SK, Wan AWA. An experimental study of the interface pressure profile during level walking of a new suspension system for lower limb amputees. Clin Biomech (Bristol, Avon). 2013;28:55-60.

[15] Wolf SI, Alimusaj M, Fradet L, Siegel J, Braatz F. Pressure characteristics at the stump/socket interface in transtibial amputees using an adaptive prosthetic foot. Clin Biomech (Bristol, Avon). 2009;24:860-5.

[16] Radcliffe CW. The biomechanics of below-knee prostheses in normal, level, bipedal walking. Artif Limbs. 1962;6:16-24.

[17] Sanders JE, Karchin A, Fergason JR, Sorenson EA. A noncontact sensor for measurement of distal residual-limb position during walking. J Rehabil Res Dev. 2006;43:509-16.

[18] Wirta RW, Golbranson FL, Mason R, Calvo K. Analysis of belowknee suspension systems: effect on gait. J Rehabil Res Dev. 1990;27:385-96.

[19] Yigiter K, Sener G, Bayar K. Comparison of the effects of patellar tendon bearing and total surface bearing sockets on prosthetic fitting and rehabilitation. Prosthet Orthot Int. 2002;26:206-12.

[20] Lilja M, Johansson T, Oberg T. Movement of the tibial end in a PTB prosthesis socket: a sagittal X-ray study of the PTB prosthesis. Prosthet Orthot Int. 1993;17:21-6.

[21] Tanner JE, Berke GM. Radiographic comparison of vertical tibial translation using two types of suspensions on a transtibial prosthesis: a case study. J Prosthet Orthot. 2001;13:14-6.

[22] Narita H, Yokogushi K, Shii S, Kakizawa M, Nosaka T. Suspension effect and dynamic evaluation of the total surface bearing (TSB) trans-tibial prosthesis: a comparison with the patellar tendon bearing (PTB) trans-tibial prosthesis. Prosthet Orthot Int. 1997;21:175-8. 